Fecha de recepción: 30 de septiembre de 2020 Fecha de evaluación: 14 de diciembre de 2020 Fecha de aprobación: 16 de diciembre de 2020

\section{Sufrimientos invisibles y crueldad normalizada: el caso de la exclusión del feminicidio en gestantes de la definición de la mortalidad materna de la OMs*}

\section{Para citar este artículo}

Bello, A. (2021). Sufrimientos invisibles y crueldad normalizada: el caso de la exclusión del feminicidio en gestantes de la definición de la mortalidad materna de la OMS. Via luris, 30, 151-167. https:// doi.org/10.37511/viaiuris.n30a8

\title{
RESUMEN
}

Este artículo problematiza el concepto de mortalidad materna de la actual Clasificación Internacional de Enfermedades de la OMS (CIE-11), con el objetivo de analizar las repercusiones que conlleva la exclusión del feminicidio en gestantes de las causales reconocidas de muerte materna. La OMS es la institución de referencia a partir de la cual se coordina el desarrollo de marcos normativos internacionales para el diseño de políticas públicas en salud; de allí la relevancia de centrar este análisis en la definición del concepto surgido en el seno de esta organización. El presente estudio se sustenta en una búsqueda sistemática de la literatura internacional publicada sobre el fenómeno del feminicidio en gestantes, a partir del análisis documental como estrategia metodológica. Se defenderá la hipótesis de que la categoría mortalidad materna propuesta por la oms invisibiliza las formas de sufrimiento que afectan principalmente a las mujeres subalternas en cada país y sobrevisibiliza las que aquejan a las mujeres más privilegiadas. Esta exclusión repercute en la normalización de una cierta economía de la crueldad, en la que el sufrimiento es canalizado -según la raza, la clase y el sexo- a través de la coordinación global en el campo de la política pública sanitaria.

* Este artículo hace parte de las conclusiones de la tesis de maestría Homicidio en mujeres gestantes: un estado del arte en clave de derechos humanos de las mujeres, para optar al título de magíster en Derecho, con profundización en Derechos Humanos y Derecho Internacional Humanitario, de la Universidad Nacional de Colombia, 2011. Las conclusiones fueron actualizadas en de la tesis de doctorado La gestión moderna del sufrimiento: genealogía del cuerpo sufriente en Colombia, desarrollada bajo la modalidad de cotutela entre la Universidad París 8 y la Universidad de Brasilia, (Periodo 2018.) Bogotá, Colombia.

** Doctora en Género de la Universidad de París 8. Posdoctoradaen Estudios Culturales de la Universidad de los Andes. Politóloga, especialista y magíster en DDHH y DIH de la Universidad Nacional de Colombia. Especialista y doctora en Bioética de la Universidad de Brasilia.Docente asistente, dedicación cátedra, de la Universidad del Tolima.Correo electrónico: bellourrego.alejandra@gmail.com. ORCID: 0000-0001-7530-2853
DOI: https://doi.org/10.37511/viaiuris.n30a8

Creative Commons Atribución-NoComercial-Compartirlgual 4.0 Internacional (CC BY-NC-SA 4.0).

\section{(c) (i) (5)}

Palabras clave

Mortalidad materna, feminicidio, gobierno del

cuerpo, derechos humanos de las mujeres, OMS interseccionalidad. 


\title{
Invisible suffering and normalized cruelty: The case of the exclusion of femicide in pregnant women from the WHO definition of Maternal Mortality
}

\author{
Alejandra del Rocío Bello Vrrego
}

\begin{abstract}
This article problematizes the concept of maternal mortality of the current WHO International Classification of Diseases (ICD-11) with the aim of analyzing the repercussions that entails the exclusion of femicide in pregnant women from the recognized causes of maternal death. The WHO is the reference institution from which the development of international normative frameworks for the design of public health policies is coordinated, hence the relevance of focusing this analysis on the definition given of the concept from this organization. This analysis is based on a systematic search of the international literature published on the phenomenon of femicide in pregnant women, on which documentary analysis is used as a methodological strategy. The hypothesis will be defended that the category of maternal mortality proposed by the WHO makes invisible the forms of suffering that mainly affect subaltern women in each country and makes visible those that afflict the most privileged women. This exclusion has an impact on the normalization of a certain economy of cruelty, in which suffering is channeled-according to race, class and sex-through global coordination in the field of public health policy.
\end{abstract}

Keywords

Maternal mortality, femicide, governance of the body, women's human rights, WHO, intersectionality. 


\title{
Sofrimento invisível e crueldade normalizada: O caso da exclusão do femicídio em mulheres grávidas da definição da OMS de Mortalidade Materna
}

\author{
Alejandra del Rocío Bello Vrrego
}

\section{RESUMO}

Este artigo problematiza o conceito de mortalidade materna na atual Classificação Internacional de Doenças da OMS (CID-11), com o objetivo de analisar as repercussões da exclusão do femicídio em mulheres grávidas das causas reconhecidas de morte materna. A OMS é a instituição de referência que coordena o desenvolvimento de marcos normativos internacionais para o desenho de políticas de saúde pública, daí a relevância de focalizar esta análise na definição do conceito dado por esta organização. Esta análise é baseada em uma busca sistemática da literatura internacional publicada sobre o fenômeno do femicídio em mulheres grávidas, utilizando a análise documental como estratégia metodológica. A hipótese será defendida de que a categoria de mortalidade materna proposta pela OMS torna invisíveis as formas de sofrimento que afetam principalmente as mulheres subalternas em cada país e torna visíveis aquelas que afligem as mulheres mais privilegiadas. Esta exclusão tem repercussões na normalização de uma certa economia de crueldade, na qual o sofrimento é canalizado-de acordo com a raça, classe e sexo-através da coordenação global no campo da política de saúde pública.

\section{Palavras-chave}

Mortalidade materna; femicídio; governança do corpo; direitos humanos da mulher; OMS; interseccionalidade. 


\title{
Souffrance invisible et cruauté normalisée : le cas de l'exclusion du féminicide chez les femmes enceintes de la définition de la mortalité maternelle de l'OMS
}

\author{
Alejandra del Rocío Bello Vrrego
}

\begin{abstract}
RÉSUMÉ
Cet article problématise le concept de mortalité maternelle dans l'actuelle Classification internationale des maladies de l'OMS (CIM-11) dans le but d'analyser les répercussions de l'exclusion du fémicide chez la femme enceinte des causes reconnues de décès maternel. L'OMS est l'institution de référence qui coordonne l'élaboration des cadres normatifs internationaux pour la conception des politiques de santé publique, d'où la pertinence de centrer cette analyse sur la définition du concept donnée par cette organisation. Cette analyse est basée sur une recherche systématique de la littérature internationale publiée sur le phénomène du fémicide chez les femmes enceintes, en utilisant l'analyse documentaire comme stratégie méthodologique. L'hypothèse sera défendue que la catégorie de mortalité maternelle proposée par l'OMS rend invisible les formes de souffrance qui affectent principalement les femmes subalternes dans chaque pays et rend visible celles qui affligent les femmes les plus privilégiées. Cette exclusion a des répercussions sur la normalisation d'une certaine économie de la cruauté, dans laquelle la souffrance est canalisée-selon la race, la classe et le sexe-par une coordination mondiale dans le domaine de la politique de santé publique.
\end{abstract}

Mots-clés

Mortalité maternelle; fémicide; gouvernance du corps; droits humains des femmes; OMs; intersectionnalité. 


\section{INTRODUCCIÓN}

Decir que la reducción de la mortalidad materna es un asunto de derechos humanos de las mujeres no necesita de mucha argumentación. Los diferentes instrumentos internacionales, regionales y nacionales de derechos humanos, así como la agenda global de los Objetivos de Desarrollo Sostenible (ODS) respaldan esta afirmación. El 97 \% de las muertes en maternas ocurre en países en vías de desarrollo, que en su casi totalidad corresponden con Estados herederos de administraciones coloniales. Esto, en sí mismo, tiene múltiples implicaciones desde el punto de vista de un análisis que tome como referencia la justicia y los derechos de las mujeres. Sin negar la pertinencia de estos enfoques, el presente artículo abordará la problematización de aquello que queda por fuera del foco de lo políticamente problemático en la definición del concepto de la mortalidad materna, a partir de la cual se desarrollan las políticas globales sobre el tema, así como las implicaciones que esto tiene en la vida de las mujeres. Concretamente, se analizará el caso de la exclusión del feminicidio en gestantes de la definición oficial.

Intentaré defender la hipótesis de que aun cuando es evidente la injusticia en la repartición de la mortalidad materna, utilizando como foco de lectura la definición oficial del concepto a partir de la Organización Mundial de la Salud (OMS), esta adquiere otra dimensión al tener en cuenta lo que dicha categoría oculta. En un primer apartado se analizará el marco institucional del gobierno global de la mortalidad materna, en el que se inserta la definición de la OMS. Luego se problematizará el fenómeno, partiendo de la revisión de la literatura que lo ha abordado. Finalmente, se argumentará que los límites de la categoría mortalidad materna normalizan una economía de la crueldad que canaliza el sufrimiento por raza, clase y sexo.

\section{Marco jurídico-institucional del gobierno global de la mortalidad maternidad}

La Organización Mundial de la Salud (OMS) es la institución de referencia de la Organización de las Naciones Unidas (ONU) en lo que respecta a la producción y exportación del discurso que construye el cuerpo sufriente. La definición de la mortalidad materna en el sistema de clasificación internacional de las enfermedades de la OMs es la referencia del
Grupo Interinstitucional de Estimaciones de Mortalidad Materna (MMEIG), compuesto por el Fondo de Población de las Naciones Unidas (FPNU), el Fondo de las Naciones Unidas para la Infancia (UNICEF), el Banco Mundial (BM) y la Organización Mundial de la Salud (OMS). El rol principal del MMEIG es construir y exportar parámetros para medir la mortalidad materna según una sola escala global, con el fin de comparar el fenómeno entre todos los países. Al definir estos parámetros, las organizaciones que componen el MMEIG construyen los pilares globales de la gestión de habitantes a través de los Estados nacionales. Esto hace que la definición de la OMS sea una pieza clave de la gestión global de la vida.

Según la ONU (2012), la salud es una condición, un resultado y un indicador del desarrollo durable, razón por la cual tres de los ocho Objetivos de Desarrollo del Milenio (ODM) establecidos en el año 2000 por esta organización estaban ligados a la salud. Dicha agenda global continuó en 2015 con los Objetivos de Desarrollo Sostenible (ODS), donde la salud conserva un lugar privilegiado: el tercer objetivo está enteramente dedicado a la salud, algunas de las metas del objetivo sobre igualdad de género se refieren a la salud de las mujeres y el objetivo sobre eliminación de la pobreza también pone en una posición relevante la salud de las poblaciones.

En el contexto de las diferentes agendas para la cooperación internacional, la OMS interviene desde 1990 en las políticas de salud de los Estados, en nombre de la cooperación para el desarrollo. Esta intervención consiste en la definición de lineamientos internacionales en salud y en la definición de las categorías médicas a partir de las cuales se articula la acción institucional en salud. Así pues, la OMS construye el cuerpo sufriente y los lineamientos para administrarlo desde una perspectiva médico-científica globalizada. La intervención en la política interna de los Estados miembros se justifica presentándose como un apoyo integral a los países con miras a reforzar sus sistemas de salud e instalar intervenciones eficaces destinadas a acelerar los progresos en política interna, con el propósito de alcanzar los ODS relativos a la salud (OMS, 2018a, 2018b).

La OMS opera en Ginebra (Suiza), a través de ejecutivxs salidxs de antiguas metrópolis y de las élites intelectuales de las antiguas colonias. Así pues, las personas cuyos privilegios son producidos por 
lógicas coloniales de larga data son aquellas que ocupan los cargos desde donde se deciden los lineamientos internacionales para las políticas en salud que adoptarán los Estados. A partir de 1990, la salud se posicionó como un indicador del nivel de desarrollo de un país, gracias a su rol dentro de los ODM y posteriormente de los ODS. El Banco Mundial (BM) y el Fondo Monetario Internacional (FMI) son las instituciones encargadas de evaluar el cumplimiento de estas agendas, y el alcance de las metas de los ODS constituye el indicador que les permite clasificar a un país en la geopolítica del desarrollo: desarrollado o en vías de desarrollo. En este sentido, dicha división define el escenario de intervención legítima de estas instancias internacionales en las políticas internas de los países Ilamados subdesarrollados.

Uno de los tres ODM ligados a la salud era la gestión global de la mortalidad materna. Actualmente, la meta 5.6 del ODS 5 obliga a garantizar los derechos sexuales y reproductivos de las mujeres, que entre otros implica el derecho a no morir durante el embarazo por causas prevenibles. Además, la meta 3.1 del ODS 3 apunta específicamente a reducir la tasa mundial de mortalidad materna a menos de 70 por cada 100.000 nacidos vivos para el año 2030 (OMS, 2020).

La retórica de lo sano y lo malsano no es un elemento nuevo en la geopolítica global. De hecho, fue un elemento determinante en la consolidación de la relación de dominación de las metrópolis sobre sus colonias. La geografía médica del siglo XIX fue fuertemente influenciada por el discurso médico ilustrado de la escuela de París, la cual a su vez lo estaba por la experiencia imperial francesa y europea en general. Según este discurso médico, los climas tropicales degeneraban la biología del animal humano al punto de producir diferencias raciales, entendidas como diferencias esenciales entre los cuerpos. En esta perspectiva, solo el clima europeo, definido como templado, era apto para mantener una biología sana (Dorlin, 2009). Considerar los cuerpos de los pueblos conquistados como esencialmente patológicos respecto a los blancos fue una parte importante en los discursos que naturalizaron las administraciones coloniales de los imperios europeos durante los siglos XIX y XX.

Definir al dominado como patológico, tomando al blanco como parámetro de lo sano, es una estrategia geopolítica que encuentra su génesis en las administraciones coloniales europeas. La salud atribuida a la raza blanca en el siglo XIX fue clave en la definición de la superioridad biológica y moral de los imperios, sobre la que se justificó la superioridad jurídica para intervenir los pueblos colonizados. Al tomar en cuenta la génesis imperial de la retórica de lo sano y lo malsano, emerge la dimensión geopolítica del gobierno global contemporáneo de la mortalidad materna.

Las agendas médicas globales en torno a este tema ubican la capacidad reproductiva de las mujeres entre los elementos que reproducen una geopolítica imperial contemporánea. Por una parte, la menor exposición a malas condiciones de vida en las mujeres privilegiadas es un parámetro de lo sano y, por ende, de lo desarrollado de un país. Por otra parte, el $97 \%$ de la mortalidad materna se concentra en antiguas colonias, debido a las malas condiciones de vida que se desprenden de una historia de despojos. En términos de cumplimiento de los ODS, ello indica la necesidad de que instituciones como el BM o el FMI intervengan con prioridad y de manera legítima en las políticas de los países en desarrollo. Este contraste, leído desde un prisma meramente sanitario, opone la salud de las mujeres de los países desarrollados a la patología de las mujeres de los países en desarrollo como argumento de la intervención de las organizaciones internacionales que perpetúan la geopolítica colonial que produce esta inequidad en primer lugar.

Sin cuestionar la etiología económica de los fallecimientos de las mujeres encinta, el objetivo de reducir en un $75 \%$ la tasa de mortalidad materna ha justificado la creación de canales institucionales entre la gestión global del cuerpo y las políticas públicas de los Estados subalternos. La categoría mortalidad materna de la OMs tiene un rol importante en justificar la injerencia directa del BM y del FMI en la gestión de los vientres, y, por lo tanto, en la reproducción de la población y de la fuerza de trabajo a una escala global. Desde esta perspectiva, podemos atribuirle a la categoría mortalidad materna dos funciones dentro del gobierno global de la vida: 1) utilizar la comparación entre cuerpos femeninos como parámetro para medir el desarrollo y así reproducir jerarquías de génesis colonial entre Estados que fueron metrópolis y Estados que surgieron de antiguas colonias, y 2) naturalizar los vientres como objetos legítimos de la gobernanza global, y 
con ello otorgar control sobre la reproducción de la vida al BM y al FMI.

El 24 de septiembre de 1973, el entonces presidente del BM, Robert McNamara, anunció que la reducción y la eliminación de la pobreza constituyen el objetivo principal de la institución; luego, se creó una línea específica de crédito con este fin Burgos (2009). La pobreza, definida ideológicamente como la consecuencia de la falta de desarrollo de un país, se posicionó como la explicación de la vulnerabilidad de los cuerpos colonizados. Esta delimitación del origen de la vulnerabilidad constituyó el marco retórico que delimitó las fronteras jurídicas de la intervención legítima de las organizaciones internacionales en los Estados subalternos. En los años noventa, la lucha contra la pobreza comenzó a ser reinterpretada a la luz del concepto de gobernabilidad aparecido en 1989. Aunque este término haya sido definido de muchas maneras, siempre se refiere a "la falta de reglas de juego, políticas y jurídicas, que posibilitarán la toma de decisiones a favor del crecimiento económico" (Burgos, 2009, p. 326). Ello acarreó una nueva etiología del sufrimiento del dominado: las instituciones que componen los Estados subalternos no tienen un marco jurídico apropiado para garantizar la integración de los países al mercado internacional, lo que explica la pobreza, la enfermedad, la muerte y el sufrimiento de sus ciudadanos. Esta etiología transforma la opresión de Ixs dominadxs en justificación para intervenir desde instituciones internacionales en la política interna de los Estados subalternos e, incluso, darles forma a sus instituciones, como en las épocas de las administraciones coloniales. Es así como la etiología ideológica de la vulnerabilidad del subalternx es usada por instituciones claramente alineadas con los intereses del mercado internacional, especialmente del mercado financiero, como el BM y el FMI.

A partir de los años setenta, la definición de la pobreza delimita los escenarios de la intervención legítima del BM y del FMI. Explicar el sufrimiento como el resultado de las disfunciones institucionales de los Estados reproduce una geopolítica colonial de dos formas: 1) el marco jurídico internacional que permite la injerencia externa en los Estados es justificado desde la retórica de la "salvación" de Ixs pobres, invisibilizando la relación entre la pobreza de los Estados en vía de desarrollo con la riqueza de los desarrollados; 2) las mejores condiciones de vida que se encuentran en los países que concentran los privilegios de la división internacional del trabajo son ideológicamente transformadas en pruebas de la superioridad moral de los países que dominan la escena geopolítica global. De hecho, los préstamos justificados en el marco de los ODM y luego de los ODS están insertos en la lucha global contra la pobreza. Estos préstamos implican la injerencia directa del BM y del FMI en los estados beneficiarios a través de consejos técnicos, la obligación de rendir cuentas anualmente, etc.

El marco institucional descrito nos permite abordar la mortalidad materna desde el prisma de su participación en la reproducción de una cierta geopolítica colonial. La lucha contra este fenómeno es entendida como la capacidad de los estados para probar el cumplimiento de metas definidas en agendas globales, que se traduce en mayores posibilidades de acceder a préstamos del FMl y el BM, con lo cual se deduce que el asunto no es solo sanitario sino también económico y político. Dada la presencia de esta temática en las agendas políticas globales, la disminución de la mortalidad materna justifica la intervención de diversas organizaciones internacionales en la institucionalidad de los países en desarrollo.

El andamiaje jurídico-institucional descrito ubica el contraste entre la salud de los cuerpos de las mujeres de los países desarrollados y la patología de las de los países en desarrollo como parámetros del desarrollo de un país, transformando así la salvación de las mujeres subalternas en la justificación del mantenimiento de lógicas geopolíticas coloniales.

\section{Lo que dice la literatura}

En la Clasificación Internacional de Enfermedades numero 10 (CIE-10) de 1990, la OMS respondió a la petición de la International Federation of Gynecology and Obstetrics (FIGO) para que incluyera el cálculo de la tasa de mortalidad materna de todos los fallecimientos, con independencia de sus causas (Costa, Ribas, Amorim \& Santos, 2002). La OMS respondió con la creación de las categorías muerte materna tardía y mortalidad ligada al embarazo. En esta clasificación, el feminicidio está incluido en la segunda categoría como una causa fortuita, vale decir, como un fenómeno "externo" al proceso del embarazo (OMS, 2012, p. 11). Lo anterior ubica la violencia como un elemento fortuito que casualmente ocurre sobre un cuerpo femenino y que 
casualmente ocurre mientras está gestando. Estas categorías crean la ilusión de inclusión, pero las muertes contabilizadas en la categoría mortalidad ligada al embarazo no entran en el cálculo de la tasa de mortalidad materna, según lo expresado por la OMS (2012). Ello significa que los efectos de la violencia en la vida de las mujeres en esta etapa vital quedan completamente excluidos del marco de intervención de los Estados y de las organizaciones internacionales. Pese a que la invisibilidad se mantiene, la ampliación en el sistema de categorías permitió problematizar el feminicidio y ponerlo en el foco de visión de investigadoras, principalmente norteamericanas. Esta es la razón por la que casi toda la literatura que explora el fenómeno ha sido producida en Estados Unidos y se concentra en los veinte años siguientes a la modificación de la definición.

Varias investigaciones estadounidenses realizadas entre 1990 y 2010 confirman que el feminicidio es una de las principales causas de mortalidad materna. Según la investigación realizada por McFarlane, Campbell, Sharps y Watson (2002) en diez ciudades de Estados Unidos, al menos el $5 \%$ de las mujeres que fueron víctimas de feminicidio estaban embarazadas. Además, encontraron que existió violencia de género durante el embarazo en el 7,8 \% de las denuncias de maltrato, el 25,8\% de los intentos de feminicidio y el $22,7 \%$ de los feminicidios perpetrados. Estas cifras evidencian que el $48 \%$ de las mujeres violentadas durante el embarazo están gravemente expuestas al feminicidio. Así mismo, entre el 4 \% y el $9 \%$ de las mujeres norteamericanas sufren violencia durante el embarazo, lo que significa que entre el $2 \%$ y el $4 \%$ de las mujeres tienen un alto riesgo de morir por feminicidio durante el embarazo. Según Bailey (2010), el problema podría ser aún más grave si se considera la falta de información sobre las causas de muerte de mujeres embarazadas.

Lo anterior muestra que la violencia durante el embarazo es un indicador de que una mujer está sobreexpuesta al feminicidio. Pero si contrastamos estas cifras con los resultados de las investigaciones norteamericanas realizadas en el mismo periodo por Cheng y Horon (2001) y por Parsons y Harper (1999), vemos que el riesgo de morir por feminicidio durante el embarazo es incluso mayor que el riesgo de morir por hipertensión; la principal causa: muerte materna, según la clasificación de la OMs. Por ejemplo, según Cheng y Horon (2010), entre 1993 y 2008, en el estado de Maryland, el feminicidio fue la principal causa de muerte materna durante el embarazo y en la mayoría de los casos ocurrió durante los primeros tres meses de gestación. Para los autores, de establecerse el embarazo como detonante de la violencia, el feminicidio se convertiría en la principal causa real de mortalidad materna directa.

La literatura que ha abordado la relación entre feminicidio y embarazo es en su mayoría latinoamericana. Estas investigaciones tomadas en consideración junto con la literatura epidemiológica norteamericana apuntan a que la violencia de género es la principal etiología del tipo de sufrimiento que conduce a la muerte a las mujeres durante su gestación y no la hipertensión. El embarazo es un momento del ciclo de vida en el que los hombres pueden sentir su masculinidad amenazada. Por eso, el cuerpo de la mujer embarazada deviene en el territorio sobre el cual los hombres ejercen su potencia para reconstruir la masculinidad que sintieron intimidada por la llegada del embarazo. Por ejemplo, según Blay (2008) y Oliveira, Carneiro y Amaral (1993), las principales causas del feminicidio en América Latina son la resistencia de las mujeres a aceptar un aborto, la amenaza al estatus de los hombres en la relación de pareja y las dudas sobre la paternidad. En la misma línea, las investigaciones de Campero, Walker, Hernández, Espinoza, Reynoso y Langer (2006), en México, y de Langer (2002), en toda América Latina y el Caribe, demostraron que en la mayoría de los casos el feminicidio en el embarazo fue provocado por la propia situación de gestación. Para Langer (2002), el feminicidio ha sido una forma de respuesta masculina recurrente, habitual e invisible a un embarazo no deseado. Sin embargo, el fenómeno no parece ser exclusivo de la región; por ejemplo, un estudio realizado por Cheng y Horon (2010) en Carolina del Norte mostró que la mayoría de los feminicidios se cometieron durante las primeras 28 semanas de gestación y otro realizado en Maryland por Parsons y Harper (1999) evidenció que fue durante las primeras 12 semanas. Ello podría ser un indicador de que el feminicidio se utilizó como método masculino de aborto en los Estados Unidos.

Las investigaciones existentes, pese a ser pocas, coinciden en visibilizar que el feminicidio es al menos una de las principales causas de muerte materna 
indirecta y que incluso puede que sea una de las principales causas directas. Sin embargo, la posibilidad de morir por feminicidio es sistemáticamente ignorada en las políticas globales de protección de las mujeres en esta etapa promovida por la OMS y desarrollada por la mayoría de los Estados miembros. Así lo demuestran Parsons y Harper (1999) al señalar que la mayoría de las víctimas de feminicidio en Carolina del Norte habían asistido regularmente a controles prenatales e incluso quienes brindaron atención médica conocían la situación de violencia en un tercio de los casos. En ellos, todas las interacciones entre la institución médica y las mujeres antes del feminicidio se habían limitado a intervenir los cuerpos para que no murieran por causas obstétricas, aun cuando era evidente que el riesgo real era morir por violencia de género.

Según la literatura, el feminicidio no está distribuido de manera uniforme en la población. Independientemente de la heterogeneidad de los enfoques y los diferentes contextos donde se han desarrollado estas investigaciones, todas ellas convergen para demostrar que las mujeres jóvenes, pobres y no blancas son las principales víctimas del feminicidio en el embarazo. En las mujeres blancas de clase media, el embarazo es un factor de protección contra la violencia de género, lo que hace que mueran principalmente por causas reconocidas. Por otro lado, el feminicidio es una causa de muerte para las poblaciones subalternas, lo que significa que los sistemas de protección del cuerpo de las embarazadas se limitan a evitar lo que mata a las mujeres privilegiadas por razones de raza y clase: mujeres blancas de clase media. De acuerdo con McFarlane, Campbell, Sharps y Watson (2002), en Estados Unidos las mujeres negras están 300 \% más expuestas al feminicidio que las mujeres blancas. De manera similar, el hecho de ser una adolescente embarazada es uno de los principales factores de riesgo para morir por feminicidio. Dado que en el país norteamericano el embarazo adolescente se concentra en las comunidades pobres, en su mayoría negras e hispanas, son las mujeres no blancas las que están más expuestas a morir por causas excluidas de los sistemas de protección estatales. Esto significa que la diferencia entre las causas obstétricas y las no obstétricas es que con este sistema de clasificación las mujeres blancas de clase media son las únicas que resultan efectivamente protegidas contra aquello que podría aquejarlas durante el embarazo, pues el despliegue biopolítico se focaliza sobre las causas reconocidas como obstétricas, que son las que podrían amenazar su vida. El resultado de esta clasificación sobre las mujeres subalternas es que aquello que puede hacerlas sufrir, enfermar y morir es dejado en un punto ciego institucional, normalizando así una exposición diferencial al sufrimiento y la muerte por razones en las que se imbrican el sexo, la raza y la clase social.

\section{Normalización de la crueldad y mortalidad materna}

El marco de inteligibilidad del cuerpo sufriente encinta de la OMS está comprendido en las categorías mortalidad materna, mortalidad materna tardía y mortalidad vinculada al embarazo ${ }^{1}$. Estas tres categorías cubren la totalidad de los fallecimientos de las mujeres hasta un año después del fin de la gestación, pero solamente la primera forma parte del marco de control global del cuerpo sufriente. A primera vista, esta clasificación constituye un paso adelante respecto a la última clasificación de las enfermedades de la OMS (CIE-11), pues permite censar todos los fallecimientos de las mujeres, independientemente de la causa de la muerte, incluso un año después del fin del embarazo. Sin embargo, la distinción entre causas obstétricas y no obstétricas, en la que esta se basa, opera a partir de una jerarquización entre la mortalidad materna -en la que se focalizan todas las políticas públicas en salud-y las que no serán prioritarias. En ese sentido, los fallecimientos catalogados como causas no obstétricas son explícitamente excluidos de la mortalidad materna y entendidos como fallecimientos vinculados al embarazo. Aunque los Estados estén obligados a censar todos los fallecimientos, en la

1 Estas categorías se complementan con los siguientes conceptos:

Muerte materna tardía, definida como el fallecimiento de una mujer resultado de causas obstétricas directas o indirectas, ocurrido más de 42 días, pero menos de un año, después del término del embarazo.

Muerte vinculada al embarazo, definida como el fallecimiento de una mujer ocurrido durante el embarazo o no más allá de los 42 días posteriores a su final, sea cual sea la causa de la muerte.

La muertes maternas se subdividen en dos grupos:

Fallecimiento por causa obstétrica directa: son los que resultan de complicaciones obstétricas (embarazo, trabajo de parto, parto y secuelas), intervenciones, omisiones, tratamientos incorrectos o un encadenamiento de eventos derivado de alguno de los factores mencionados.

Fallecimiento por causa obstétrica indirecta: son los que resultan de una enfermedad preexistente o de una afección aparecida durante el embarazo, sin que se deba a causas obstétricas directas, pero que fue agravada por los efectos fisiológicos del embarazo. 
práctica esta distinción conlleva la autorización e incluso la animación a focalizarlos dentro de los que se contabilizan como mortalidad materna. Lo más grave es que gracias a este sistema de clasificación, el feminicidio está excluido de todos los mecanismos de presión sobre los Estados mencionados en el primer apartado. Ahora bien, tal como será demostrado, esta exclusión constituye una forma invisible de gobernar los cuerpos femeninos, caracterizada por la naturalización de la exposición a la crueldad dosificada en función de diversas categorías de diferencias, especialmente la raza y la clase.

En la última clasificación de las enfermedades de la OMS (CIE-11), la mortalidad materna está definida exclusivamente como "el fallecimiento de una mujer ocurrido durante su embarazo o durante los 42 días posteriores a su fin, sean cuales sean la duración y la localización, por cualquier motivo determinado o agravado por el embarazo o los cuidados que motivó, pero ni accidental ni fortuito" (OMS, 2009, p. 179). Esta definición traza las fronteras del marco de visión de la medicina. Considerar el feminicidio como una causa no obstétrica acarrea su expulsión del terreno de la intervención médica, dejándolo fuera del terreno en el que el BM y el FMI obligan a los Estados a que intervengan eficazmente. Así pues, el efecto directo de la clasificación de este fenómeno como una causa no obstétrica es sacarlo del marco a partir del cual las instituciones que gobiernan la vida definen el sufrimiento políticamente relevante.

Varias investigaciones realizadas en diferentes países de forma independiente coinciden en señalar que el feminicidio es una de las primeras causas de mortalidad en todas las mujeres entre 13 y 49 años, y al menos una de las cinco causas principales de mortalidad en el grupo de mujeres en gestación, si no la primera (Cheng \& Horon, 2010; Fino, 2007; Parsons \& Harper, 1999). La creciente visibilidad del fenómeno coincide con la ampliación de la perspectiva acerca de la mortalidad materna introducida por la OMS en la CIE-10, lo que muestra la centralidad del rol de esta organización en la definición de la frontera entre el sufrimiento "visible" y el "invisible". La literatura internacional fue producida en su mayoría entre 1990 y 2010 en Estados Unidos. El informe sobre el sistema de vigilancia de la mortalidad materna de 2005 del Center for Disease Control and Prevention (CDC) fue determinante en este proceso, pues este señala que el $20 \%$ de las mujeres que mueren durante el embarazo son asesinadas, lo que convierte al homicidio en la principal causa de muerte de las mujeres embarazadas en Estados Unidos. Otras investigaciones anteriores y posteriores a dicho informe coinciden en este porcentaje y también en otros aspectos. Por ejemplo, el fenómeno se concentraba en las mujeres jóvenes afroamericanas; la no declaración del embarazo en el censo oficial de los fallecimientos por feminicidio es enorme; durante el embarazo una mujer está al menos tres veces más expuesta a ser violentada o asesinada; las mujeres menores de 20 años son las más expuestas y el embarazo a esta edad es más común en los contextos de marginalización económica a la que son relegadas la mayoría de las latinas y afroamericanas; las afroamericanas son las más expuestas a morir por feminicidio durante el embarazo; la mayoría de los homicidios son cometidos con armas de fuego y vivir en una zona donde hay una amplia circulación de estas armas aumenta su exposición; por último, hay más armas en las zonas de habitación de la gente no blanca debido a su integración en el lado ilegal de la economía global (Bello-Urrego, 2011). Las escasas investigaciones que abordan el fenómeno en otros países llegan a conclusiones equivalentes ${ }^{2}$ a las del CDC, principalmente respecto a la amplitud del fenómeno y a la mayor exposición de las jóvenes no blancas que viven en contextos de marginalización económica. Ello indica que no se trata de una particularidad de Estados Unidos, sino más bien de un fenómeno ligado a lógicas globales ${ }^{3}$.

El número de investigaciones que explora el feminicidio es ínfimo si lo comparamos con la literatura científica que aborda las causas incluidas en la tasa de mortalidad materna. Esta falta de exploración prueba la efectividad del marco de construcción del cuerpo sufriente encinta de la OMS para fijar las fronteras del campo de visión del cuerpo sufriente. El vínculo entre la definición de la OMS y el quinto ODM también contribuye a esta invisibilidad, en tanto incentiva a los países en vías de desarroIlo a alcanzar los ODM para poder mostrar cifras

2 Estudios de la República Checa, Bangladesh, Rusia, Chile, India, Sri Lanka y Pakistán fueron recopilados por Maria Bruyn (2003). Respecto a la literatura latinoamericana, ver Bello-Urrego (2011).

3 Aunque la mayoría de las investigaciones no abordan la relación entre embarazo y feminicidio, las que existen, en su mayoría latinoamericanas, indican que la violencia de género no se radicaliza de manera fortuita durante el embarazo de manera fortuita, sino debido a este. Ver al respecto: Oliveira, Carneiro y Amaral (1993); Blay (2008); Campero, Walker, Hernández, Espinoza, Reynoso y Langer (2006), y Langer (2002) 
descendientes de mortalidad materna al BM y al FMI, ya que de ello depende el acceso a la cooperación internacional. Esto puede animar a los Estados a estimular exclusivamente las investigaciones acerca de las causas reconocidas por la OMS, sobre las que serán evaluados, y también a mantener fuera del campo de visión los factores que podrían afectar los indicadores previstos en los informes.

Entre 1998 y 2004, el feminicidio y el suicidio ${ }^{4}$ durante el embarazo fueron la principal causa de fallecimiento en el conjunto de las mujeres en edad reproductiva ${ }^{5}$. En Colombia, el feminicidio de las mujeres embarazadas se reparte en el territorio siguiendo la misma distribución racial que la mortalidad materna por causas reconocidas. Según Fino (2007), en el Chocó, la Amazonia y la Orinoquia existe la mayor mortalidad materna del país, llegando a ser cinco veces más elevada que el promedio nacional. En estas regiones también hay un alto porcentaje de población perteneciente a pueblos indígenas y negros. En otras regiones donde la población no blanca es mayoritaria y donde históricamente ha habido poca o nula presencia estatal, el feminicidio es la principal causa de muerte

4 La investigación analiza el feminicidio y el suicidio como fenómenos conexos. Aunque esté de acuerdo con la existencia de un estrecho vínculo entre ambos y con el propósito de respetar la delimitación del tema de la tesis, solo incluyo en mi análisis los datos sobre el feminicidio. Sin embargo, el caso del suicidio en las mujeres encinta también ilustraría la mayoría de los argumentes presentados en esta sección. Putumayo, Guainía y Caquetá son las regiones donde más se suicidan las mujeres encinta (Fino, 2007). El Putumayo es, además, la región donde se constata a la vez la mayor cantidad de suicidios y la segunda mayor cantidad de feminicidios. Son regiones con una gran presencia de pueblos indígenas. En la cosmovisión de los pueblos de la Amazonia, el suicidio es la respuesta más digna frente a la exposición sistemática a la crueldad. Históricamente, los pueblos indígenas de esta región han sido integrados en la economía capitalista del lado ilegal de la división internacional del trabajo. De ahí que estos pueblos hayan sido gestionados como recursos por actores armados y no como poblaciones para el Estado (ver capítulo II). Por ejemplo, luego de la intensificación del conflicto armado en Colombia durante los años 1990, los paramilitares pudieron consolidar, en los años 2000, su control territorial de esta zona estratégica para la explotación minera y el narcotráfico (Centro Nacional de Memoria Histórica, 2016). Tal como lo demuestra el estudio de Ginna Coral, psiquiatra del pueblo Camëntsá del Putumayo, el suicidio es una estrategia de resistencia frente a la negación del acceso al bienestar para los pueblos indígenas, algo estructural a la división internacional del trabajo. En este contexto, los embarazos no deseados son uno de los elementos identificados por la investigadora como factor detonante del suicidio en las mujeres de los pueblos indígenas (Coral, 2015).

5 Categoría médica utilizada para designar el grupo de mujeres entre 13 y 49 años de edad. de mujeres en gestación, mientras que a nivel nacional esta es la tercera causa. Ejemplo de ello son el Guaviare, Putumayo y Caquetá. Otro elemento en común a todas estas regiones es que se trata de territorios fuertemente afectados por el conflicto armado y por el tráfico de drogas.

Una investigadora del Instituto Nacional de Salud ${ }^{6}$ (INS) intentó consultar los expedientes jurídicos respecto a los feminicidios más allá de las cifras, pero no fue posible debido a límites institucionales del Ministerio de Justicia. Sin embargo, se le autorizó consultar un expediente jurídico elegido aleatoriamente. Se trataba del caso de una mujer embarazada de seis meses, asesinada por su compañero, un paramilitar (Fino, 2016). Esta mujer vivía en Córdoba, región que en el momento de los hechos estaba siendo invadida por un grupo paramilitar. Su cuerpo y el de su bebé fueron descuartizados, una estrategia de asesinato típica de los paramilitares, utilizada en todos los territorios de Colombia. Este caso se inscribe en el marco del conflicto armado, aunque el combatiente haya actuado en su propio interés. Hay que tener en cuenta que la masculinidad militarista depende más que las otras de la continua reafirmación del poder a través del consumo de cuerpos femeninos ${ }^{7}$. Esta capacidad depredadora, que va más allá de la participación directa del hombre en el grupo armado, ha sido probada por el hecho de que después del proceso de paz de 2006 -que debía desmovilizar la organización paramilitar de las Autodefensas Unidas de Colombia (AUC)-, aumentó enormemente la violencia clasificada como "intrafamiliar" en Colombia. Por otra parte, el hecho de que las tres regiones con la mayor cantidad de feminicidios sean zonas bajo control paramilitar ilustra el vínculo entre destrucción del cuerpo femenino y presencia paramilitar en un territorio.

Desde principios del siglo $\mathrm{xx}$, el paramilitarismo formó parte de las estrategias de conquista y de integración de los territorios en la economía global para provecho de las élites coloniales. Según Bello-Urrego (2021), se trata de la racionalización de la violencia ejercida sin ningún límite contra las comunidades cuya humanidad no es reconocida por la mirada moderna, y que por lo tanto no están

6 Este órgano independiente se encargaba de la producción de datos epidemiológicos para el Ministerio de la Salud.

7 Ver el concepto de mandato de masculinidad de Rita Segato (2016). 
sometidas a través del disciplinamiento institucional, sino a través del ejercicio directo de la crueldad. Los territorios controlados por estas milicias siempre han sido económicamente estratégicos y, al mismo tiempo, albergan a las poblaciones dejadas al margen en los procesos de construcción de la nación ${ }^{8}$. Según Romero (2007), las milicias paramilitares han sido eslabones clave en la cadena de elaboración y distribución de productos de la economía legal global: aceite de palma, varios productos de explotación minera, bananos, etc. Aunque estos productos no sean ilícitos, su cadena de producción depende del control ilegal de los territorios y de sus habitantes. Además, las organizaciones paramilitares también han estado implicadas en la economía de productos ilegales. Según Mantilla (2012), la organización paramilitar Autodefensas Unidas de Colombia (AUC), surgida en el contexto neoliberal de finales del siglo $x x-y$ luego los diferentes grupos armados reconstituidos a pesar del proceso de paz del 2006- están directamente implicados en la explotación minera ilegal y el narcotráfico. Estas son actividades que permiten la concentración de grandes cantidades de dinero, necesarias para participar en actividades del lado legal del mercado transnacional, una vez el dinero es blanqueado.

El control territorial de los paramilitares siempre se ha valido de la depredación de los cuerpos femeninos. En efecto, la destrucción de las mujeres es inseparable de la conquista de los territorios y de la economía global que depende de ello. El exterminio de las embarazadas ejemplifica esta lógica. En el ya clásico libro La Violencia en Colombia, de Germán Guzmán Campos, Orlando Fals Borda y Eduardo Umaña Luna (1962), se documenta cómo los chulavitas y los pájaros, milicias paramilitares colombianas de principios del siglo XX, asesinaban públicamente y con métodos muy crueles a mujeres embarazadas de las comunidades consideradas enemigas del proyecto político del Partido Conservador, considerando este hecho como el símbolo de la "destrucción" de la "semilla" del enemigo. Dichos grupos estaban vinculados a los intereses de los sectores económicos cuyo poder dependía

8 Los paramilitares están estrechamente ligados a la explotación de los territorios a través de medios ilegales y parainstitucionales, al servicio de empresas legales de tipo crianza industrial de vacas, empresas transnacionales mineras, así como empresas transnacionales de la cultura del banano y la palma africana, etc. Estos grupos también están ligados al narcotráfico y a la explotación minera ilegal (CNMH, 2011, p. 72). del latifundio. En los años noventa y dos mil, las AUC continuaron esta lógica de destrucción simbólica del enemigo a través de la aniquilación concreta de las mujeres embarazadas. Esa forma de feminicidio es una expresión extrema de la destrucción del cuerpo femenino que se despliega en la conquista de los territorios por parte de actores parainstitucionales ${ }^{9}$.

De la misma manera que en Colombia, en otros países los feminicidios de las mujeres embarazadas se concentran en los territorios donde la economía ilegal es predominante o está muy presente, tal como en los barrios afroamericanos y latinos en Estados Unidos o el estado de Morelos en México. Esta economía acarrea disputas de territorios donde la única vía para mostrar el poderío es la violencia directa. El funcionamiento del lado ilegal de la economía se basa en la demostración de fuerza y no en normas institucionalizadas, y la crueldad hacia los cuerpos femeninos subalternos se inserta en estas lógicas, pues estos constituyen el principal territorio disponible para el hombre no blanco donde demostrar su potencia. Según Bello-Urrego (2021), aunque toda masculinidad implica la depredación de otros seres, la división colonial de la vida convierte la depredación a través de la burocracia del lado legal del mercado en una expresión del privilegio del hombre blanco, mientras que la masculinización militarista que hace funcionar el lado ilegal de este mismo mercado está reservada al no-blanco. A pesar de esto, las investigaciones que abordan el fenómeno del feminicidio en gestantes solo han indagado por la violencia de género ejercida en el contexto de la pareja, lo que deja fuera del campo de visión la relación entre la depredación de los cuerpos femeninos y el funcionamiento normal de la economía capitalista.

En los informes de la Comisión Nacional de Memoria Histórica sobre la violencia contra las mujeres ejercida por las AUC hay varios casos de mujeres torturadas y/o asesinadas públicamente porque estaban embarazadas. Estos son cinco entre los más representativos de los diferentes aspectos del fenómeno: una niña encinta violada brutalmente por el jefe de la facción, con el objetivo de disciplinar al subalterno responsable del embarazo (CNMH, 2011); una joven de 18 años empalada públicamente porque se sospechaba que estaba embarazada de

\footnotetext{
9 En lo que concierne a este vínculo entre depredación del cuerpo femenino y funcionamiento normal de la economía, ver Segato (2016).
} 
un guerrillero (CNMH, 2009); una mujer de la que se sospechaba que era guerrillera, violada ferozmente con el objetivo de hacerla abortar frente a su compañero, sospechoso también de ser guerrillero, bajo el argumento de que la rebelión es hereditaria $(\mathrm{CNMH}$, 2016); una mujer que utilizó con éxito el argumento de estar embarazada para evitar una agresión $(\mathrm{CNMH}$, 2011), y una mujer que fue la única sobreviviente de un grupo de mujeres condenadas a muerte gracias al hecho de estar embarazada (CNMH, 2009).

En los dos primeros casos, el embarazo fue concebido como una "marca" que indica la propiedad de un hombre sobre una mujer. Este elemento también se presenta en el tercer caso, pero en este la mujer es considerada indeseable. En el segundo y en el tercer caso, la mujer embarazada fue blanco de la destrucción material y simbólica de la capacidad reproductora del enemigo. Sin embargo, en el cuarto y en el quinto caso, la capacidad reproductora expresada por el embarazo pudo haber jugado un rol protector. En efecto, los cuerpos hiperfeminizados por la gestación devienen objeto de una protección especial o, por el contrario, objetos particularmente valiosos para ejercer la crueldad en sentido "pedagógico". A estos últimos corresponden las mujeres acusadas por los paramilitares de ser el enemigo o de "reproducir al enemigo". La focalización de las agresiones sobre el vientre, el feto y la vagina en la destrucción de los cuerpos de las mujeres embarazadas muestra que también son atacadas por el hecho de ser mujeres, por la gestación y por ser identificadas como reproductoras del grupo enemigo. Las que encarnan la lejanía de la expectativa paramilitar acerca de las conductas aceptables son construidas como cuerpos poco valiosos a través de la crueldad, pero el grupo que se reproduce a través de ellas también es "desvalorizado". Estas últimas son las que le sirven al grupo armado para disciplinar a la comunidad respecto al comportamiento aceptable en una mujer. Ellas no mueren fortuitamente estando embarazadas, al contrario, esa es la razón de su muerte. Para los paramilitares, lo que importa es el mensaje que la crueldad sobre estas mujeres envía a las comunidades que viven en los territorios bajo su control.

Las pocas investigaciones que abordan cualitativamente el vínculo entre el asesinato y el embarazo se limitan al análisis de la violencia en el contexto de la pareja. Oliveira, Carneiro y Amaral (1993) ejemplifican los hallazgos de la literatura internacional $^{10}$ : un hombre casado mató a una niña menor de edad con quien sostenía una relación de abuso, después de que ella rechazara abortar, y otro mató a su esposa porque no encontraba trabajo para mantenerla. En estos casos se ejerció la crueldad sobre las embarazadas, reduciéndolas a propiedades de las que se puede disponer en función del interés del autor del feminicidio. Los principales agresores son esposos, novios, antiguos compañeros, entre otros, para quienes el embarazo significa una pérdida de control. Las investigaciones existentes muestran que el feminicidio es una respuesta masculina común frente a los embarazos no deseados ${ }^{11}$. Aunque el fenómeno haya sido visibilizado en estudios latinoamericanos, esto no parece ser una particularidad de la región. Por ejemplo, según la literatura epidemiológica estadounidense, el feminicidio tiene lugar principalmente durante los primeros meses del embarazo, lo que podría indicar que se trata de una respuesta a embarazos no deseados.

El análisis conjunto de los casos abordados en la literatura acerca de la violencia contra las mujeres embarazadas y de aquellos documentados por los informes para la memoria histórica en Colombia muestra que el estatus de objeto de los cuerpos femeninos no existe previamente a la crueldad. Al contrario, es la crueldad la que reduce, en mayor o menor grado, a los cuerpos femeninos a objetos sobre los que un otro construye su propio poder. Esta dinámica es mucho más explícita en el caso del conflicto armado colombiano, pero también está presente en los casos dados dentro del contexto de la pareja ${ }^{12}$. Es una lógica patriarcal que constituye un continuum entre los casos, en los cuales las mujeres fueron asesinadas por el hecho de ser mujeres. No obstante, hay una diferencia entre aquellas cuya muerte se enmarca en el lado institucional y legal de la economía y aquellas cuya muerte se enmarca en el funcionamiento del lado ilegal y parainstitucional. Aunque en todos los casos las mujeres mueren por

10 La búsqueda en las bases de datos fue realizada mediante los motores de búsqueda internacionales en cuatro lenguas: portugués, inglés, francés y español. La mayoría de la literatura accesible con este método es latinoamericana, por lo que se deduce que el enfoque del presente estudio es probablemente más frecuente en esta región.

11 Ver Bello-Urrego (2011); Campero, Walker, Hernández, Espinoza, Reynoso y Langer (2006), y Langer (2002).

12 Utilizo esta categoría para hacer referencia a las categorías utilizadas más comúnmente por la literatura internacional. 
el hecho de ser mujeres, también mueren debido a su inserción en la economía global. Según la literatura científica internacional revisada, los datos oficiales sobre la mortalidad materna en Colombia y los informes de memoria histórica del $\mathrm{CNMH}$, las mujeres víctimas de feminicidio durante el embarazo son casi exclusivamente mujeres no blancas que viven en zonas donde predomina el lado ilegal del capitalismo. Esto implica que no todos los cuerpos femeninos están igualmente expuestos a la muerte por el hecho de ser mujeres, y que la exclusión de la violencia de la definición de mortalidad materna es un ejercicio de crueldad "racializante". El feminicidio de estas mujeres, así como la exclusión del fenómeno de la categoría de la OMS, son actos de crueldad que niegan la dignidad tanto de las mujeres afectadas como del grupo social al que pertenecen.

Las exclusiones generadas por el marco de la OMS al abordar el cuerpo de las mujeres embarazadas muestran una gestión racializante de los cuerpos, que reproduce desde el vientre diferencias profundas entre los sujetos. Cuando una causa de muerte es clasificada dentro de la mortalidad materna, los Estados están obligados a intervenir con políticas cuya efectividad será verificada por el BM y el FMI. Esta focalización obliga al Estado a intervenir en las causas de mortalidad materna a las que están expuestas las mujeres más privilegiadas. Al contrario, todas las mujeres víctimas de feminicidio son clasificadas como muertes asociadas al embarazo. Por eso, al ser excluidos del censo de la mortalidad materna, estos fallecimientos son transformados en cifras no prioritarias ni para el Estado ni para las organizaciones internacionales. Así pues, no solo no se interviene para cuidar a estas mujeres, sino que además se les expone -aún más que a otras mujeres- a formas normalizadas de crueldad. Las regiones que tienen la población no blanca más importante de Colombia son también las zonas donde las mujeres mueren más debido a causas clasificadas como asociadas al embarazo que por las causas reconocidas como mortalidad materna. De 10 fallecimientos reconocidos como "vinculados al embarazo", 4 eran feminicidios y 3 suicidios, lo que implica que 7 de 10 fallecimientos corresponden a muertes violentas de mujeres embarazadas, que debido a esta categoría son transformadas en hechos "fortuitos"; por ende, se ubican fuera del campo de visión y de intervención institucional.
De otra parte, la distinción entre causas obstétricas y no obstétricas crea una frontera entre el cuerpo sufriente y el cuerpo normal. La exclusión de la violencia de género del cuerpo sufriente construye indirectamente la crueldad como un elemento intrínseco al cuerpo femenino. Esto permite intervenir el cuerpo de forma médica sin problematizar su inserción en las relaciones de dominación que lo feminizan, lo cual constituye de por sí un ejercicio de naturalización de las relaciones de poder que producen los cuerpos. Este ejercicio de poder no afecta de la misma manera a todos los cuerpos, pues la distribución de los feminicidios indica que las mujeres en posiciones sociales subalternas están más expuestas a la violencia que se queda fuera del marco de la intervención médica. Así pues, la exclusión de la violencia de género de la definición de la mortalidad materna institucionaliza una canalización racializante del sufrimiento hacia los grupos subalternos.

La construcción del cuerpo sufriente es un ejemplo de la manera como se normaliza una cierta repartición de la crueldad al definir la frontera entre el sufrimiento susceptible de intervención institucional y el que no. Este ejercicio de delimitación es en sí mismo un ejercicio de crueldad, pues se naturaliza la exposición estructural de ciertos cuerpos al sufrimiento, la enfermedad y la muerte.

La categoría muerte vinculada al embarazo invisibiliza lo que mata a las mujeres no blancas y pobres durante el embarazo. En ese sentido, esta categorización médica puede ser abordada como un mecanismo efectivo de naturalización de la exposición diferenciada a la crueldad de los cuerpos femeninos según matrices de dominación.

\section{CONCLUSIONES}

Al dejar la violencia de género fuera de los límites de la intervención médica, el marco hegemónico de inteligibilidad del cuerpo gestante sitúa al feminicidio fuera de los límites de la acción de las instituciones globales y nacionales que gobiernan el sufrimiento. Mediante la exclusión de la violencia de género como posible causa de mortalidad materna, la OMS está produciendo activamente una ilusión óptica sobre las causas reales de la muerte de las mujeres en esta etapa de la vida. Esta ilusión es en realidad una etiología ideológica del sufrimiento que 
naturaliza la violencia de género como un elemento normal en la vida de las mujeres.

La exposición al sufrimiento tiene mucho que ver con las matrices de dominación que clasifican los cuerpos por raza, clase y sexo, entre otras categorías de diferencia. No todas las mujeres están igualmente expuestas; las más privilegiadas están casi exclusivamente expuestas a causas obstétricas. Son las mujeres subalternas las que están sistemáticamente más expuestas a formas de sufrimiento "invisible", al morir más por causas no obstétricas, como la violencia. La focalización de recursos en las causas obstétricas favorece a las mujeres ya privilegiadas a costa de exponer estructuralmente a las mujeres subalternas. Así, la aparente neutralidad del discurso médico-científico que define el gobierno institucional de la maternidad en realidad está profundamente imbricado con la reproducción de matrices de dominación patriarcales, racistas y clasistas. 


\section{REFERENCIAS}

Bailey, B. A. (2010). Partner Violence During Pregnancy: Prevalence, Effects, Screening, and Management. International Journal of Women's Health, 2, 183-197. Recuperado de https://www.ncbi.nlm.nih.gov/pmc/articles/ PMC2971723/

Bello-Urrego, A. (2011). Homicidio en mujeres gestantes: un estado del arte en clave de derechos humanos de las mujeres. Tesis de maestría Universidad Nacional de Colombia, Bogotá, Colombia.

Bello-Urrego, A. (2021). El giro a la derecha en América Latina. La crueldad y el gobierno de los cuerpos-Otros en la etapa neoliberal del capital. Estudios Políticos, 60, 118-143. Recuperado de https://doi.org/10.17533/ udea.espo.n60a06

Blay, E. A. (2008). Assassinato de mulheres e direitos humanos. Editora 34.

Burgos, G. (2009) Estado de derecho y globalización: el banco Mundial y las reformas institucionales en América Latina. ILSA

Campero, L., Walker, D., Hernández, B., Espinoza, H., Reynoso, S. \& Langer, A. (2006). La contribución de la violencia a la mortalidad materna en Morelos, México. Salud Pública de México, 48, s297-s306. Recuperado de https://doi.org/10.1590/S003636342006000800010

Centro Nacional de Memoria Histórica. (2009). La masacre de El Salado. Esa guerra no era nuestra. Organización Internacional para las Migraciones (OIM-Misión Colombia).

Centro Nacional de Memoria Histórica. (2011). Mujeres y guerra. Víctimas y resistentes en el Caribe colombiano. Taurus. Recuperado de https://centrodememoriahistorica. gov.co/wp-content/uploads/2020/01/
Mujeres-y-Guerra.-V\%C3\%ADctimas-y-Resistentes-en-el-Caribe-Colombiano.pdf

Centro Nacional de Memoria Histórica. (2016). El placer. Mujeres, coca y guerra en el bajo Putumayo. Recuperado de https://centrodememoriahistorica.gov.co/wp-content/ uploads/2020/10/El-Placer-2020-web.pdf

Chang, Berg, Saltzman y Herndon (2005) y Parsons y Harper. (2005) Homicide: A Leading Cause of Injury Deaths Among Pregnant and Postpartum Women in the United States, 1991-1999. Am J Public Health. 2005 March; 95(3): 471-477. Recuperado de https://www. ncbi.nlm.nih.gov/pmc/articles/PMC1449204/

Cheng, D. \& Horon, I. L. (2001). Enhanced Surveillance for Pregnancy-Associated MortalityMaryland, 1993-1998. JAMA, 285(11), 14551459. Recuperado de https://jamanetwork. com/journals/jama/fullarticle/193666

Cheng, D. \& Horon, I. L. (2010). Intimate-Partner Homicide Among Pregnant and Postpartum Women. Obstetrics and Gynecology, 115(6), 1181-1186. Recuperado de https:// doi.org/10.1097/AOG.0b013e3181de0194

Coral Palchucán, G. A. (2015). Narrativas sobre la vida, la muerte y la conducta suicida en indígenas estudiantes de la Universidad Nacional de Colombia-Sede Bogotá-pertenecientes al pueblo Camëntsá. 2013-2014. Tesis de especialización Universidad Nacional de Colombia, Bogotá, Colombia. Recuperado de https://repositorio.unal.edu.co/bitstream/handle/unal/54265/43972014.2015. pdf?sequence $=1 \&$ isAllowed $=y$

Dorlin, E. (2009). La Matrice de la Race Généalogie Sexuelle et Coloniale de la Nation Française. La Découverte.

Fino Sandoval, D. E. (2007). Mortalidad relacionada con el embarazo. Colombia 1985-2005: Orinoquia, Amazonia y Chocó. Tesis de 
especialización Universidad de Antioquia, Medellín, Colombia.

Fino Sandoval, D. E. (2016). Mujeres: retos a la formación médica y praxis en salud. Reflexiones sobre género, interculturalidad, decolonialidad y derechos humanos. Tesis de maestría Universidad Pedagógica Nacional, Bogotá, Colombia. Recuperado de http://repository.pedagogica.edu.co/bitstream/handle/20.500.12209/7816/TO-21379. pdf? sequence $=1 \&$ isAllowed $=y$

Guzmán Campos, G., Fals Borda, O. \& Umaña, E. (1962). La Violencia en Colombia. Estudio de un proceso social. Bogotá: Tercer Mundo.

Langer, A. (2002). El embarazo no deseado: impacto sobre la salud y la sociedad en América Latina y el Caribe. Public Health, 11(3), 192-204. Recuperado de http://www.scielosp.org/

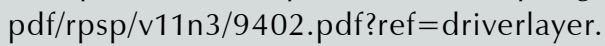
com/web

Mantilla Valbuena, S. (2012). Economía y conflicto armado en Colombia: los efectos de la globalización en la transformación de la guerra. Latinoamérica, 55, 35-73. Recuperado de http://www.scielo.org.mx/pdf/latinoam/n55/ n55a3.pdf

McFarlane, J., Campbell, J. C., Sharps, P. \& Watson, K. (2002). Abuse During Pregnancy and Femicide: Urgent Implications for Women's Health. Obstetrics and Gynecology, 100(1), 27-36.

Oliveira Menicucci, E., Carneiro, V. \& Amaral, L. (1993). Violência Conjugal na Gravidez. Revista de Estudios Feministas, 165(1), 62-65.

Organización de las Naciones Unidas (ONU). (2012). Naciones Unidas Rio+20. El futuro que queremos. https://www.un.org/ga/search/view_ doc.asp?symbol=A/RES/66/288\&Lang $=S$

Organización Mundial de la Salud (OMS). (2012). Guía de la OMS para la aplicación de la CIE-10 a las muertes ocurridas durante el embarazo, parto y puerperio: CIE-MM. Recuperado de https://www.paho.org/clap/dmdocuments/ CIE10MM.pdf

Organización Mundial de la Salud (OMS). (2018a). Hacia un Plan de acción mundial a favor de una vida sana y bienestar para todos. Recuperado de https://apps.who.int/iris/bitstream/ handle/10665/312005/WHO-DCO-2018.3spa.pdf

Organización Mundial de la Salud (OMS). (2018b). Objetivos de Desarrollo del Milenio (ODM). Recuperado de https://www.who.int/es/ news-room/fact-sheets/detail/millenniumdevelopment-goals-(mdgs)

Organización Mundial de la Salud (OMS). (2020). Objetivos de Desarrollo Sostenible: Metas. Recuperado de https://unstats.un.org/sdgs/ report/2020/The-Sustainable-DevelopmentGoals-Report-2020_Spanish.pdf

Organización Panamericana de la Salud, Organización Mundial de la Salud (2017). Lineamientos básicos para el análisis de la mortalidad. Tomado de: https://iris.paho.org/bitstream/ handle/10665.2/34492/9789275319819-spa. pdf? sequence $=7$

Parsons, L. H. \& Harper, M. A. (1999). Violent Maternal Deaths in North Carolina. Obstetrics and Gynecology, 94(6), 990-993.

Romero, M. (Edit.). (2007). Parapolítica. La ruta de la expansión paramilitar y los acuerdos políticos. CEREC; Corporación Nuevo Arco Iris.

Segato, R. L. (2016). La guerra contra las mujeres. Traficantes de Sueños. 